\title{
Lyman-alpha radiative transfer during the epoch of reionization: contribution to $21-\mathrm{cm}$ signal fluctuations
}

\begin{abstract}
B. Semelin, F. Combes, and S. Baek
LERMA, Observatoire de Paris, UPMC, CNRS, 61 Av. de l'Observatoire, 75014 Paris, France

e-mail: benoit. semelin@obspm.fr

Received 29 May 2007 / Accepted 31 July 2007

ABSTRACT

During the epoch of reionization, Ly- $\alpha$ photons emitted by the first stars can couple the neutral hydrogen spin temperature to the kinetic gas temperature, providing an opportunity to observe the gas in emission or absorption in the 21-cm line. Given the bright foregrounds, it is particularly important to determine the fluctuation signature of the signal precisely, so as to be able to extract it by its correlation power. LICORICE is a Monte-Carlo radiative transfer code, coupled to the dynamics via an adaptative Tree-SPH code. We present here the Ly- $\alpha$ part of the implementation and validate it through three classical tests. Unlike previous works, we do not assume that $P_{\alpha}$, the number of scatterings of Ly- $\alpha$ photons per atom per second, is proportional to the Ly- $\alpha$ background flux, but take the scatterings in the Ly- $\alpha$ line wings into account. The latter have the effect of steepening the radial profile of $P_{\alpha}$ around each source, and re-inforce the contrast of the fluctuations. In the particular geometry of cosmic filaments of baryonic matter, Ly $-\alpha$ photons are scattered out of the filament, and the large-scale structure of $P_{\alpha}$ is significantly anisotropic. This could have strong implications for the possible detection of the 21-cm signal.
\end{abstract}

Key words. methods: N-body simulations - radiative transfer - method: numerical - galaxies: intergalactic medium cosmology: large-scale structure of Universe

\section{Introduction}

The epoch of reionization (EoR) extends from the time when the first sources form as a result of the nonlinear growth of primordial density fluctuations in a fully neutral universe $(z \sim 20)$ to the moment when the intergalactic medium is fully reionized under the effect of UV radiations emitted by the sources $(z \sim 6)$. This simple picture, however, hides a number of uncertainties. At this time, there are only two observational constraints on the EoR. The first comes from the Gunn-Peterson effect in the absorption spectrum of high redshift quasars. Indeed the transmitted flux drops sharply as a small neutral fraction appears toward high redshifts, which implies an ionization fraction $x_{\mathrm{HI}}<10^{-4}$ at $z<5.5$ (Fan et al. 2006). The second constraint is set by the Thomson scattering of CMB photons by the free electrons produced during the EoR. The corresponding optical depth, $\tau=0.09 \pm 0.03$ (Spergel et al. 2007) implies that the intergalactic medium is already significantly reionized at $z=11$. In the next decade, we will learn a lot more by direct observation of the redshifted $21-\mathrm{cm}$ emission from the neutral IGM during the EoR. Such instruments as LOFAR, PAST, or MWA will be able to probe the statistical properties of the $21-\mathrm{cm}$ signal, while SKA will be able to make a full tomography of the IGM up to $z \sim 11$. See Carilli et al. (2004) and Carilli (2006) for detailed prospects.

In the last decade, a lot of work has been done, both theoretical and numerical, to predict the properties of the $21-\mathrm{cm}$ emission and better optimize the design of the future instruments. Madau et al. (1997) and Tozzi et al. (2000) have presented the first theoretical models of $21-\mathrm{cm}$ emission. The signal can be seen either in emission or in absorption against the CMB, depending on the spin temperature of the neutral hydrogen.
Interaction with $\mathrm{CMB}$ photons couples the spin temperature to the CMB temperature in less than $10^{5}$ years during the EoR, which would make the signal undetectable. Fortunately, two other processes tend to couple the spin temperature to the gas kinetic temperature instead. The first is the excitation of the hyperfine transition through collisions with electrons or other hydrogen atoms (see Nusser 2005; Kuhlen et al. 2006, for numerical simulations), which is, however, only efficient in overdense regions (baryonic $\delta \rho / \rho>5 /[(1+z) / 20]^{2}$ ). The second is the pumping of the $21-\mathrm{cm}$ transition by Ly- $\alpha$ photons (Wouthuysen-Field effect, Wouthuysen 1952; Field 1959), which requires a threshold value for the local Ly- $\alpha$ flux to be effective. Consequently, the value of the kinetic temperature of the gas relative to the CMB temperature is crucial for determining the $21-\mathrm{cm}$ emission brightness temperature.

During the EoR, the gas is cooling down due to the expansion of the universe faster than the CMB, but is heated by hydrodynamical shocks from structure formation, by X-ray from pop-III star or quasars and, to a much lesser extent, by Ly- $\alpha$ photons (see e.g., Furlanetto et al. 2006). Simple analytical models are now available that take various source types and formation history into account (Furlanetto 2006). They usually predict that the signal can be seen in absorption early on, then later on in emission. The prediction of the typical amplitude for the differential brightness temperature is a few $10 \mathrm{mK}$. Analytical models cannot, however, take the full complexity of the 3D inhomogeneous IGM into account. Indeed, both numerical simulations including the dynamics of structure formation and, usually as a posttreatment, a full 3D radiative transfer are required.

Dynamical simulations of structure formation have a long history, but cosmological radiative transfer simulations are a more recent field of investigation. Simulations showed that the 
ionized bubbles around the first sources are not spherical: indeed the ionization fronts propagate fast in the void and more slowly in the high-density filaments (see Abel et al. 1999, for the first simulation). The geometry of reionization is now studied in large simulation boxes $(\sim 100 \mathrm{Mpc})$ to get a statistical sample of ionized bubbles of various sizes (Iliev et al. 2006a; McQuinn et al. 2007). However, in such large boxes, even with very high resolution simulations, small-scale density structures, also called minihaloes, are not resolved and are often included through a simple clumping factor. The global effect of minihaloes is to slow down reionization by consuming photons during their photoevaporation. But a simple, uniform clumping factor may be insufficient for modeling the role of minihaloes, and more detailed studies have been performed (Ciardi et al. 2006; Iliev et al. 2005; and Shapiro et al. 2006 for the impact on the $21-\mathrm{cm}$ signal). Using the density field from the dynamical simulations and the ionization fraction and gas temperature from the radiative transfer simulations, it is possible to produce $21-\mathrm{cm}$ emission maps.

A number of authors provide predictions for different aspects of the 21-cm signal: the emission map at a given redshift, the average signal as a function of redshift, or the signal power as a function of the angular scale. These quantities were first predicted from rather small simulation boxes $(\sim 10 \mathrm{Mpc})$ limiting the angular information to $\theta<10$ arcmin (Ciardi \& Madau 2003; Gnedin \& Shaver 2004; Furlanetto et al. 2004; Valdes et al. 2006). Recently, predictions from larger simulation boxes $(\sim 100 \mathrm{Mpc})$ became available (Mellema et al. 2006; Iliev et al. 2007). It is a fact that the predictions, in particular the duration and intensity of the absorption phase, crucially depend on the modeling of the sources. But other factors have the potential to alter these predictions. Indeed, all predictions from simulations, at this time, assume a uniform efficiency for the WouthuysenField effect. However, Barkana \& Loeb (2005) or Furlanetto et al. (2006a) recognize that fluctuations in the local Ly- $\alpha$ flux can produce additional fluctuations in the brightness temperature. An accurate quantitative modeling of these fluctuations is important. Indeed, the $21 \mathrm{~cm}$ signal will be difficult to detect due to brighter foregrounds: but the unique signature of its brightness fluctuations will make the extraction possible. Barkana \& Loeb used simplified analytic models (neglecting radiative transfer effects on the local Ly- $\alpha$ flux) to compute this contribution. Our goal in this paper is to investigate how computing the full radiative transfer in the Ly- $\alpha$ line in a cosmological, inhomogeneous medium can modify the picture presented by these authors. The $21 \mathrm{~cm}$ signal will be difficult to detect, due to brighter foregrounds, and the unique character of its brightness fluctuations is a signature that will make its extraction possible. It is therefore crucial to predict those fluctuations in more details.

Although Monte-Carlo simulations of Ly- $\alpha$ transfer have a long history, starting with Avery \& House (1968), only recently has the computing power become sufficient for tackling the case of a 3D inhomogeneous medium with kinematics, without restrictions on the optical thickness regime (Ahn et al. 2001). Several authors have now developed similar codes to simulate the Ly- $\alpha$ emission from high-redshift galaxies (Zheng \& Miralda-Escudé 2002; Cantalupo et al. 2005; Dijkstra et al. 2006; Verhamme et al. 2006; Tasitsiomi 2006).

In this paper, we present the implementation and validation of the Ly- $\alpha$ radiative transfer in LICORICE, a dynamicsradiative transfer code, with special emphasis on the treatment of large Hubble flows, which is specific to the EoR applications. This work is part of the SKADS ${ }^{1}$ effort (DS2-T1 task) to produce

\footnotetext{
${ }^{1}$ http://www.skads-eu.org
}

simulated emission maps that can be used to optimize the design of the future SKA telescope.

In Sect. 2, we present which physics of the Ly- $\alpha$ transfer is included in LICORICE and justify why some aspects of the physics do not need to be included. In Sect. 3 we explain some aspects of the algorithms and acceleration schemes implemented in the code. Three validation tests are presented in Sect. 4, comparing the outputs of LICORICE to either analytic solutions or standard numerical results. Finally, in Sect. 5, we apply the code to some typical EoR situation to investigate possible fluctuations in the Wouthuysen-Field effect.

\section{The code: LICORICE}

LICORICE consists of three parts. A TreeSPH code with multiphase modeling of the gas computes the dynamics of structure formation (Semelin \& Combes 2002). A continuum radiative transfer part is added to compute reionization. This part uses a Monte-Carlo approach and is similar to CRASH (Maselli et al. 2003). It has the advantage over CRASH of using an adaptative grid. LICORICE is currently participating in the second part of the Cosmological Radiative Transfer Comparison Project (see Iliev et al. 2006b, for the first part of the project). The third part is the Ly- $\alpha$ radiative transfer, which is described in this paper.

\subsection{Physics of the $L y$ - $\alpha$ radiative transfer}

LICORICE implements a Monte-Carlo approach to radiative transfer by propagating photons on an adaptative grid. Consequently, we describe the physics of Ly- $\alpha$ radiative transfer from the point of view of a photon traveling through the simulation box.

\subsubsection{Computing the optical depth}

A photon propagating through neutral hydrogen has a probability $P(\tau)=\mathrm{e}^{-\tau}$ of not being scattered after traveling through an optical depth $\tau$ from its emission point. We only consider Ly- $\alpha$ scattering, so the optical depth can be computed as:

$\tau=\int_{0}^{l} \int_{-\infty}^{+\infty} \mathrm{d} s \mathrm{~d} u_{\|} n_{\mathrm{HI}} p\left(u_{\|}\right) \sigma\left(v\left(1-\frac{v_{\|}^{\text {macro }}+u_{\|}}{c}\right)\right)$,

where $v$ is the photon frequency in the global rest frame and $n_{\mathrm{HI}}$ the local number density of neutral hydrogen. Also, $u_{\|}$is the scattering atom velocity along the incoming photon's direction in the moving reference frame of the fluid, $p\left(u_{\|}\right)$the normalized probability distribution for $u_{\|}, v_{\|}^{\text {macro }}$ the gas macroscopic velocity along the incoming photon's direction in the global rest frame, and $c$ the speed of light. Finally, $\sigma\left(v^{\prime}\right)$ is the Ly- $\alpha$ scattering cross section of a photon with frequency $v^{\prime}$ in the atom rest frame.

The function $p\left(u_{\|}\right)$usually results from the thermal distribution of the atoms velocity:

$p\left(u_{\|}\right)=\frac{1}{\sqrt{\pi} v_{\text {th }}} \exp \left(-\frac{u_{\|}^{2}}{v_{\text {th }}^{2}}\right)$ with $v_{\text {th }}=\sqrt{\frac{2 k_{\mathrm{B}} T}{m_{\mathrm{p}}}}$.

Some astrophysical systems have significant velocity gradiants on scales below the best possible resolution of the simulations. In these cases it may be relevant to model the small-scale velocity contribution by adding a turbulent component to the thermal velocity dispersion. We did not include any turbulent contribution in this paper. 
The exact expression of the Ly- $\alpha$ scattering cross section is given in Peebles (1993). It is well-approximated by the usual Lorentzian profile:

$\sigma(v)=f_{12} \frac{\pi e^{2}}{m_{\mathrm{e}} c} \frac{\Delta v_{L} / 2 \pi}{\left(v-v_{0}\right)^{2}+\left(\Delta v_{L} / 2\right)^{2}}$,

where $f_{12}=0.4162$ is the Ly- $\alpha$ oscillator strength, $v_{0}=2.466 \times$ $10^{15} \mathrm{~Hz}$ is the line center frequency, and $\Delta v_{\mathrm{L}}=9.936 \times 10^{7} \mathrm{~Hz}$ is the natural line width.

We introduce the dimensionless parameters $x$, the relative frequency shift, and $b$, the natural to Doppler line width ratio:

$x=\frac{v-v_{0}}{\Delta v_{\mathrm{D}}} \quad$ with $\quad \Delta v_{\mathrm{D}}=\frac{v_{\text {th }}}{c} v_{0}$,

$b=\frac{\Delta v_{\mathrm{L}}}{2 \Delta v_{\mathrm{D}}}$.

Using these notations, we can write the optical depth increment in the local rest frame of the gas $\left(v_{\|}^{\text {macro }}=0\right)$ as

$\mathrm{d} \tau=\mathrm{d} s n_{\mathrm{HI}} \frac{f_{12} \sqrt{\pi} e^{2}}{m_{\mathrm{e}} c \Delta v_{\mathrm{D}}} H(b, x)$,

or in cgs units

$\mathrm{d} \tau=\mathrm{d} s n_{\mathrm{HI}} 6.87 \times 10^{-14}\left(\frac{T}{10^{4}}\right)^{-\frac{1}{2}} H(b, x)$,

where $H$ is the Voigt function defined as

$H(b, x)=\frac{b}{\pi} \int_{-\infty}^{+\infty} \frac{\mathrm{e}^{-y^{2}}}{(x-y)^{2}+b^{2}} \mathrm{~d} y$.

To compute the Voigt function, we either use the analytic fit given by Tasitsiomi (2006) or, in cosmological situations where the Hubble flow on a scale of the order of the simulation spatial resolution produces a frequency shift larger than the Ly- $\alpha$ line width (see Sect. 2.1.3), we use the simple approximation

$H(x, b) \simeq \max \left(\mathrm{e}^{-x^{2}}, \frac{b}{\sqrt{\pi} x^{2}}\right)$.

\subsubsection{Effect of the $n=2$ state splitting, dust, and deuterium.}

A Ly- $\alpha$ photon can excite a hydrogen atom from the $1 S_{1 / 2}$ ground state to either the $2 P_{1 / 2}$ or the $2 P_{3 / 2}$ state. As discussed by Tasitsiomi (2006), the splitting between these two $n=2$ states is small: only $10 \mathrm{GHz}$ or $1 \mathrm{~km} \mathrm{~s}^{-1}$. If the thermal velocity dispersion is much larger than $\sim 1 \mathrm{~km} \mathrm{~s}^{-1}$ (i.e. $T>100 \mathrm{~K}$ ), the level splitting is washed out from the radiation spectrum after just one scattering. In the case of the Ly- $\alpha$ background radiation during the EoR, the gas temperature drops to $\sim 30 \mathrm{~K}$. However the optical depth as the photon redshifts from one side to the other of the Ly- $\alpha$ line is very high: $\sim 8 \times 10^{5}$ for the average gas density of the universe $\left(\Omega_{\mathrm{b}}=0.045\right)$ at $z=9.5$ in a standard cosmological model. Thus the photon will scatter off thermal atoms many times and still the splitting will be washed out. Consequently, we did not distinguish between the two $2 P$ states in this paper.

Another issue is the possible reshuffling from $2 P$ to $2 S$ through collisions with free protons or electrons. An atom cannot be excited directly to the $2 S$ state because of the dipole selection rule. But if the $2 P \rightarrow 2 S$ transition is induced by a collision, then the atom will de-excite through the emission of 2 continuum photons losing the Ly- $\alpha$ photon. Tasitsiomi (2006, see Eq. (26)) computed the probability that an atom in the $2 P$ state will reshuffle to $2 S$ before it de-excites normally by emitting a Ly- $\alpha$ photon, $p \sim 8.5 \times 10^{-13} n_{\mathrm{p}}\left(\frac{T}{10^{4}}\right)^{-0.17}$, where $n_{\mathrm{p}}$ is the proton number density. What can we expect during the EoR? First, we are interested in the Ly- $\alpha$ background in the cold, neutral region of the universe, thus $n_{\mathrm{p}} \ll 1$. Moreover, even if we assume a non negligible ionization fraction, $p \sim 5 \times 10^{-13}$ at $z \sim 10$ for $T=50 \mathrm{~K}$ and for the critical density of the universe with $\Omega_{b}=0.045$. This probability must be compared with the average number of scatterings a photon undergoes as it redshifts through the Ly- $\alpha$ line, which is similar to the optical depth: $\sim 10^{6}$. We see that only a fraction of $5 \times 10^{-7}$ of the Ly- $\alpha$ photons will be degraded into 2 continuum photons before they redshift out of the line, so we conclude that this process is not relevant during the EoR.

Dust is usually a factor in Ly- $\alpha$ transfer simulations since it absorbs Ly- $\alpha$ photons. Hansen \& Oh (2006) study the effect of dust absorption in a multiphase medium and show that photons can escape such a medium, while they would be absorbed in a more homogeneous single-phase medium, for the same total HI column density. In the cosmological context we are working in $(z>6)$, there are no observations to help us constrain the dust abundance and distribution. We can assume that, during the EoR, dust may be found only around the sources and that the IGM is completely dust free. Under this assumption, the effect of dust on the Ly- $\alpha$ flux can be modeled with a simple escape-fraction coefficient. Furthermore, if this coefficient is assumed to be independent of the source, dust will not have any effect on the Ly- $\alpha$ flux fluctuations that are the focus of this paper. Therefore, we did not include the effect of dust.

Dijkstra et al. (2006) show that the presence of deuterium with an abundance $[\mathrm{D} / \mathrm{H}]=3 \times 10^{-5}$ leaves a clear imprint on the spectrum emerging from a uniform sphere of gas with a central source and a total optical depth $\tau=7.3 \times 10^{5}$. Is deuterium relevant to the Ly- $\alpha$ flux during the EoR? The deuterium line center is $82 \mathrm{~km} \mathrm{~s}^{-1}$ blueward of the hydrogen line. This is equivalent to the redshift of a photon traveling $\sim 0.5$ comoving Mpc at $z=9.5$, so the first answer is that deuterium may have an effect on the Ly- $\alpha$ flux fluctuations only on small scales ( $<1$ Mpc comoving). However, the total optical depth for deuterium through the Ly$\alpha$ line is $\tau \sim 20$ for an abundance $[\mathrm{D} / \mathrm{H}]=2 \times 10^{-5}$, and the optical depth in the wing of the Hydrogen Ly- $\alpha$ line, as the photon redshifts from far in the blue to the center of the deuterium line happens to also be $\tau \sim 20$. This means that, while the photon will indeed scatter a few times in the deuterium line, it will also have been scattered in the hydrogen line wing several times before it reaches the frequency range where deuterium scattering dominates (a few $10 \mathrm{~km} \mathrm{~s}^{-1}$ around the line center). We conclude that the presence of deuterium is unlikely to affect the Ly- $\alpha$ flux fluctuations noticeably. This does not mean that the effect of deuterium on Ly- $\alpha$ radiation cannot be observed during the EoR. Indeed, in the case of an ionizing bubble around a source with a sharp ionization front, the continuum spectrum of the source will show a Gunn-Peterson trough with a step at the bubble redshift. Deuterium should create a secondary small step.

\subsubsection{Dealing with large Hubble flows}

In $z \sim 10$ cosmological simulations, the Ly- $\alpha$ thermal line width is equivalent to the Hubble-flow redshift over only a few 10 comoving kpc. This scale is usually (much) smaller than the size of cells in simulations, so we must be careful when we compute the optical depth: using a single comoving frequency for the photons 
throughout a cell would result in photons flowing through the line core without feeling it. We must actually compute an integral along the path of the photon, with a redshifting comoving frequency, to obtain the correct optical depth. If we consider that the expansion velocity between any two points of the same cell is non-relativistic $\left(\frac{v_{\mathrm{H}}}{c} \sim 0.01\right.$ for 20 comoving Mpc at $\left.z \sim 10\right)$, the computation gets easier. Let $v_{\text {in }}$ be the comoving frequency and $x_{\text {in }}$ the local rest frame value of $x$ when the photon enters the cell:

$x_{\text {in }}=\frac{v_{i n}\left(1-\frac{v^{\text {macro }} \cdot \boldsymbol{k}}{c}\right)-v_{0}}{\Delta v_{D}}$,

where $\boldsymbol{v}^{\text {macro }}$ is the macroscopic velocity of the gas (uniform inside the cell), and $\boldsymbol{k}$ the direction of the photon. Then, at a given point inside the cell defined by the vector $r \boldsymbol{k}$ from the entering point of the photon, the comoving frequency is

$v=\frac{a\left(v_{\text {in }}\right)}{a(v)} v_{\text {in }} \sim \frac{v_{\text {in }}}{1+\frac{\mathrm{H} r}{c}} \sim v_{\text {in }}\left(1-\frac{\mathrm{H} r}{c}\right)$,

where $\mathrm{H}$ is the Hubble constant at the simulation redshift, and $a$ the expansion factor. We neglect any variation of $\mathrm{H}$ during the photon travel and consider non-relativistic expansion velocities. For non-relativistic macroscopic velocities of the gas, the corresponding value of $x$ writes as

$x=x_{\text {in }}-\frac{\mathrm{H} r}{c} \frac{v_{\text {in }}}{\Delta v_{\mathrm{D}}}$

As a result, we linearized the relation between $r$, the current path length inside the cell, and the current local rest-frame value of the $x$ variable. In this approximation, noting $x_{\text {out }}$ as the value of $x$ when the photon exits the cell, computing the optical depth reduces to computing the integral Voigt function $H_{\text {int }}\left(x_{\text {out }}\right)$, with the following definition for the $H_{\text {int }}$ function:

$H_{\text {int }}(x)=\int_{x_{\text {in }}}^{x} H\left(x^{\prime}, b\right) \mathrm{d} x^{\prime}$.

However, in the Monte-Carlo method, to find the location of a scattering event, we need to solve the equation $H_{\text {int }}(x)=A$, where $A$ is a constant. This is simplified if we can provide an analytic expression for $H_{\text {int }}(x)$, with an explicit inverse function. This is the case if we use the simple approximation of $H(x, a)$ given in Eq. (9). It involves the $\operatorname{erf}(x)$ function for which we are using an approximation that has an explicit inverse function.

Modeling the effects of expansion only by a redshift computed from a radial dilatation is the usual approximation for Ly- $\alpha$ radiative transfer codes. Other effects of the variation in the expansion factor $a$, such as variation in the average density during the photon flight time, are usually ignored. However, let us emphasize that adding the expansion velocity to the other types of velocities (macro or microscopic) to compute Doppler shifts, either during scattering events or for computing local rest frame values of $x$, works only if all velocities are non-relativistic. However, we want to study the Ly- $\alpha$ flux during the EoR; and at $z \sim 10$, a photon emitted just below the Ly- $\beta$ frequency will travel $\sim 350$ comoving $\mathrm{Mpc}$ and be redshifted to Ly- $\alpha$ by a Hubble flow velocity such that $v_{\mathrm{H}} / c \sim \frac{\delta v}{v} \sim \frac{\delta a}{a} \sim 0.15$. In this case, the second-order errors in computing the redshift are not completely negligible. But, what is more important, neglecting the variations in $a$ along the photons' path, in computing gas densities for example, produces first-order errors in $\frac{\delta a}{a}$. Consequently, we should limit ourselves to $\sim 100$ comoving $\mathrm{Mpc}$ boxes. In this work, we do neglect variations in $a$, except for the cosmological redshift, and in most cases we use simulation boxes smaller than $\sim 30$ comoving Mpc. The full effect of expansion will be introduced in the future to handle larger boxes.

\subsection{Scattering off hydrogen atoms}

In an expanding universe, the natural variable is the comoving frame frequency of the photon. However, as long as the Hubble flow velocities are non-relativistic, we can use the frequency in the rest frame of the zero-coordinate point of the simulation, hereafter named global frame, and treat the expansion as a simple radial dilatation with a $\boldsymbol{v}_{\mathrm{H}}=\mathrm{H} \boldsymbol{r}$ velocity field to be added to the peculiar velocities.

In the rest frame of the atom, we consider the scattering to be resonant. The effect of the recoil, which would change the frequency of the photon by transferring part of its momentum to the atom, has been shown to be negligible in astrophysical situations by several authors (Zheng \& Miralda-Escudé 2002; Tasitsiomi 2006). In the global frame, however, the frequency $v$ of the photon will change due to the various contributions to the atoms velocity.

There are three main contributions to the atom velocity: the thermal motion, the macroscopic peculiar motion and the Hubble flow. When the photon scatters off an atom, we first compute the frequency in the atom rest frame:

$v_{\text {atom }}=v\left(1-\left(\frac{\boldsymbol{v}_{\mathrm{H}}+\boldsymbol{v}^{\text {macro }}+\boldsymbol{u}}{c}\right) \cdot \boldsymbol{k}_{i}\right)$,

where $\boldsymbol{u}$ is the thermal velocity of the atom, and $\boldsymbol{k}_{i}$ the direction of the incoming photon. Let us split $\boldsymbol{u}$ into $\boldsymbol{u}_{\|}$, the component parallel to the incoming photon direction, and $\boldsymbol{u}_{\perp}$, the perpendicular component. $\frac{\boldsymbol{u}_{\|}}{v_{\mathrm{th}}}$ obeys the distribution:

$P_{1}(y)=\frac{b}{\pi H(b, x)} \frac{\mathrm{e}^{-y^{2}}}{(x-y)^{2}+b^{2}}$,

while each of the 2 components of $\boldsymbol{u}_{\perp}$ obeys

$P_{2}(y)=\frac{1}{\sqrt{\pi} v_{\text {th }}} \mathrm{e}^{-\frac{y^{2}}{v_{\mathrm{th}}^{2}}}$.

Then we compute the direction of the photon after scattering. As described by Tasitsiomi (2006) the scattering phase function depends on the excitation state and on whether the photon scatters in the wing or in the core of the line. Several authors (see e.g. Cantalupo et al. 2005; Verhamme et al. 2006 ) have shown that, for high optical depth media, the shape of the phase function does not alter the results of the simulation. In this work, we use isotropic scattering. With the new direction of the photon, we then recompute the frequency in the global frame using a non-relativistic Doppler effect.

\section{Ly- $\alpha$ radiative transfer: numerical methods}

\subsection{Gas density and velocity field}

LICORICE is meant to use results from dynamical simulations realized with the TreeSPH part of the code (Semelin \& Combes 2002). It uses the same Tree structure as the dynamical code to build an adaptative grid. The grid is built in such a way that each cell contains between 1 and $N_{\max }$ gas particles. Values from 1 to 30 are commonly used for $N_{\max }$. The density and velocity fields are then interpolated from the particle distribution. The 
cells limit the resolution of the simulation only by having uniform dynamical properties. Ly- $\alpha$ transfer inside one cell is still computed exactly under the assumption that the expansion velocity on the scale of the cell is non-relativistic.

\subsection{Monte-Carlo method}

Using the Monte-Carlo approach, we send individual photon from the source and follow them from scattering to scattering and from grid cell to grid cell until they exit the simulation box. After one event (emission or scattering), the algorithm is the following:

- Step 1: compute the photon global frame frequency either from the scattering atom rest frame frequency or from the source spectrum.

- Step 2: draw the new photon direction (isotropically in this work).

- Step 3: draw a variable $p$ from a uniform distribution between $[0,1]$. The photon will travel an optical depth $\tau=$ $-\ln (p)$ to the next scattering event.

- Step 4: increment optical depth with current cell contribution. Determine if scattering occurs in this cell: if yes, go to step 5; if no, pass on to next cell and repeat step 4.

- Step 5: draw scattering atom thermal velocity and compute frequency in the scattering atom rest frame. Go back to step 1.

\subsection{Acceleration scheme}

To generate random variable following Eq. (15) distribution, we use the method by Zheng \& Miralda-Escudé (2002). These authors introduce a parameter $u_{0}$ for which an optimal value is needed. We use the following empirical fit:

$u_{0}=1.85-\log (b) / 6.73+\ln (\ln (x))$ for $x>3$,

and $u_{0}=0$ otherwise. This has been determined from a systematic numerical optimization and works well for $10^{-4}<b<10^{-1}$. For high values of $x$, quite common in cosmological simulations, drawing a value from $P_{1}$ is still slow, even with the Zheng \& Miralda-Escudé method. In this case, however, scattering will most likely occur in the wing of the line. Consequently, for $x>10$, we revert to $u_{0}=0$ but truncate the distribution to the limited range $[-3,3]$.

We use the core-skipping acceleration scheme; see Avery \& House (1968) or Ahn et al. (2002) for the first applications. In this scheme, we choose a core value $x_{\mathrm{c}}$ for the variable $x$. In the regime $x<x_{\mathrm{c}}$, the medium must be thick. If so, the photon will scatter many times over a very short distance. Only when $x$ gets larger than $x_{\mathrm{c}}$ (scattering in the wing by a fast moving atom) will the medium become transparent and the photon will travel a long distance. The idea is to ignore the insignificant core scatterings: every time the photon enters the core $\left(x<x_{\mathrm{c}}\right)$, it leaves again immediately by scattering off an atom with a thermal velocity $u_{\text {at }}>x_{\mathrm{c}} v_{\text {th }}$. We use the detailed prescriptions given by Tasitsiomi (2006) on choosing $x_{\mathrm{c}}$ as a function of $b \tau_{0}$, where $b$ is defined in Eq. (5) and $\tau_{0}$ is the optical depth at the line center. The coreskipping scheme works well when the expected output of the code is an emerging spectrum. We show that it also works for computing the fluctuations of the local scattering rate.

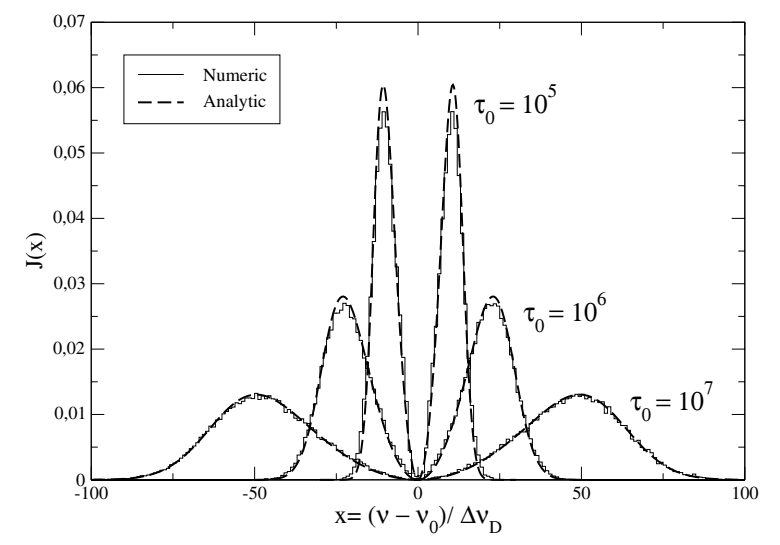

Fig. 1. Emerging spectrum for a uniform and spherical cloud of gas at $T=10 \mathrm{~K}$ and several values of $\tau_{0}$, the total optical depth at line center. Numerical histograms are computed with $10^{5}$ photons. The analytic solution is given in Dijkstra et al. (2006).

\section{Validation tests}

Since LICORICE is a complex, multipurpose code, we make certain to validate each part separately. We present validation tests for the Ly- $\alpha$ part here, comparing our results to analytical solutions or standard numerical setups.

\subsection{Static homogeneous sphere: emerging spectrum}

A classical validation test for Ly- $\alpha$ codes is the emerging spectrum for a monochromatic source in the middle plane of a static homogeneous slab of gas. The main reason is that Neufeld (1990) gives an analytic solution for the emerging spectrum in the case of an extremely thick system. However, Dijkstra et al. (2006) provide a new analytic expression in the case of a uniform spherical cloud of gas:

$J(x)=\frac{\sqrt{\pi}}{\sqrt{24} a \tau_{0}}\left[\frac{x^{2}}{1+\cosh \left(\sqrt{\frac{2 \pi^{3}}{27}} \frac{\left|x^{3}\right|}{a \tau_{0}}\right)}\right]$.

Since this geometry is more relevant in a cosmological context, we present results for this case (we also performed the Neufeld test and found good agreement). Figure 1 shows the comparison between numerical and analytic spectra for a static spherical cloud of gas with temperature $T=10 \mathrm{~K}$ and optical depths at line center from center to edge equal to $10^{5}, 10^{6}$, and $10^{7}$. For each run, $10^{5}$ photons were injected at the center of the cloud with frequency $v_{0}$. The emerging spectrum $J(x)$ was obtained by computing a normalized histogram of the frequencies of the photons as they leave the cloud. The agreement is good in all cases, although it gets better and better as $\tau_{0}$ increases. Indeed in the case $\tau_{0}=10^{5}$, we have $a \tau_{0}=1500$, which is close to the lower limit for an extremely thick medium.

\subsection{Expanding homogeneous sphere: emerging spectrum}

The second test is also becoming a classic. It was first performed by Zheng \& Miralda-Escudé (2002), followed by several authors such as Verhamme et al. (2006) who present especially detailed results. The system is a uniform sphere of gas expanding (or contracting) with a Hubble-like velocity field: the radial velocity is proportional to the radius. We used the same physical condition as Zheng \& Miralda-Escudé (2002): a temperature of $20000 \mathrm{~K}$ 


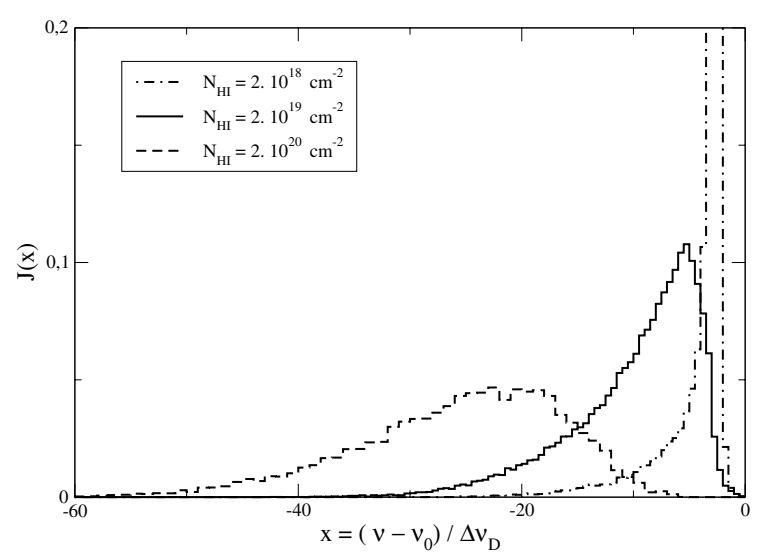

Fig. 2. Emerging spectrum for a spherical, uniform, and expanding cloud of gas at $T=20000 \mathrm{~K}$ and several values of $N_{\mathrm{HI}}$, the column density from the center to the edge of the cloud. The emission is at Ly$\alpha$ frequency from the central point of the cloud. The radial velocity of the gas is proportional to the radius and equals $200 \mathrm{~km} \mathrm{~s}^{-1}$ at the edge of the cloud. The peak blueward of the Ly- $\alpha$ frequency is completely suppressed by the expansion.

and a radial velocity of $200 \mathrm{~km} \mathrm{~s}^{-1}$ at the edge of the cloud. We used three different column densities from the center to the edge of the cloud: $N_{\mathrm{H}}$ equals $2 \times 10^{18} \mathrm{~cm}^{-2}, 2 \times 10^{19} \mathrm{~cm}^{-2}$ or $2 \times 10^{20} \mathrm{~cm}^{-2}$ (that is $\tau_{0}=8.3 \times 10^{4}, 8.3 \times 10^{5}$, or $8.3 \times 10^{7}$ ). We did not run the tests for a contracting cloud, and we only considered a central point source emitting at Ly- $\alpha$ frequency, not the case of uniform emissivity. An expanding cloud with a central point source is the most relevant to the EoR. The emerging spectra are shown in Fig. 2. We find very similar results to Zheng \& Miralda-Escudé (2002) or Verhamme et al. (2006). The peak blueward of the Ly- $\alpha$ frequency is completely suppressed by the expansion (not shown in Fig. 2). For the case of a contracting cloud, the red peak would be suppressed

\subsection{Expanding homogeneous sphere at $T=0 \mathrm{~K}$ : mean intensity field}

Our last validation test relies on an analytic solution given by Loeb \& Rybicki (1999). The setup is again an expanding uniform hydrogen medium with a central monochromatic source at Ly- $\alpha$ frequency. The velocity field is a Hubble flow again, but this time, the gas temperature is $T=0 \mathrm{~K}$. Loeb \& Rybicki introduced the dimensionless variable $\tilde{v}=\frac{v_{0}-v}{v_{\star}}$, where $v_{\star}$ is the comoving frequency shift from Ly- $\alpha$ at which the optical depth to infinity equals 1 , and $\tilde{r}=\frac{r}{r_{\star}}$, where $r_{\star}$ is the proper radius at which the Doppler shift from the source due to the Hubble expansion equals $v_{\star}$. Loeb \& Rybicki give an analytic expression for the corresponding dimensionless mean intensity $\tilde{J}$, valid in the diffusion regime:

$\tilde{J}(\tilde{r}, \tilde{v})=\frac{1}{4 \pi}\left(\frac{9}{4 \pi \tilde{v}^{3}}\right)^{\frac{3}{2}} \exp \left[-\frac{9 \tilde{r}^{2}}{4 \tilde{v}^{3}}\right]$.

Loeb \& Rybicki (1999) show the comparison between the analytic solution and the numerical solution given by a dedicated Monte-Carlo code. Tasitsiomi (2006) runs this test with his general purpose code. Our results are presented in Figs. 3 and 4. They are very similar to those of Loeb and Rybicki and Tasitsiomi: the numerical results are close to the analytic solution where the diffusion regime is valid. However, where photons enter the free-streaming regime $\left(\tilde{r}_{0}=1\right.$ in Fig. 3 and $\log \tilde{v}_{0}=0.5$

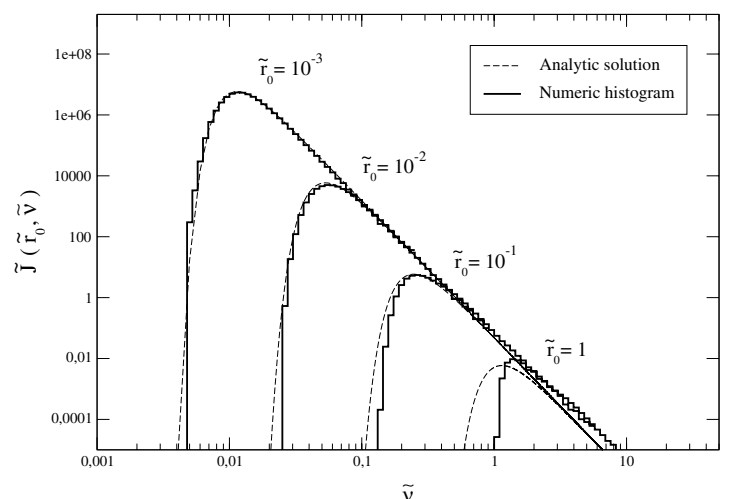

Fig. 3. Mean intensity spectra at various radii for a Ly- $\alpha$ monochromatic source in a uniform expanding medium at $T=0 \mathrm{~K}$. The numerical result is compared to the analytic solution given in the diffusion regime by Loeb \& Rybicki (1999).

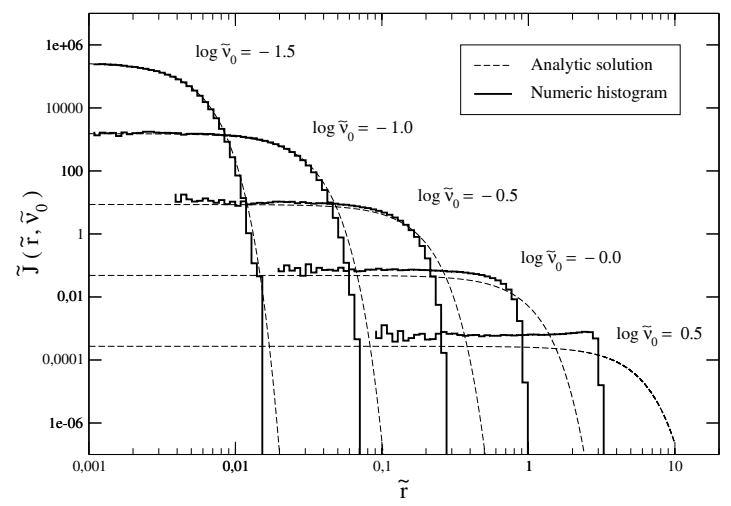

Fig. 4. Mean intensity profile at various frequencies for a $\mathrm{Ly}-\alpha$ monochromatic source in a uniform expanding medium at $T=0 \mathrm{~K}$. The numerical result is compared to the analytic solution given, in the diffusion regime, by Loeb \& Rybicki (1999).

in Fig. 4), the numerical solution diverges from the analytic solution, which becomes invalid.

Here are some details on how we ran this test. We used a temperature $T=2 \mathrm{~K}$ for computing the optical depth, which is singular for $T=0 \mathrm{~K}$, but we used a zero thermal velocity for the atoms in all scattering events. We used a simulation box holding a sphere of gas of radius $10 r_{\star}$. Since the temperature is not zero in all respects, it reintroduces a dimension into the problem. Very close to the source the thermal speed is higher than the expansion velocity and the numerical behavior should diverge from the analytic solution. For reference, we used a proper expansion velocity of $200 \mathrm{~km} \mathrm{~s}^{-1}$ at $r_{\star}$. In our setup, the thermal and expansion velocities similar for $r \sim 10^{-3} r_{\star}$.

The radii range covered by this test is quite wide, from $10^{-3} r_{\star}$ to $10 r_{\star}$. Using $10^{12}$ cells for the radiative transfer grid is not really an option. Actually, we used only $16^{3}$ cells, but we took advantage of the integral scheme described in Eqs. (12) and (13) for computing the optical depth exactly between two points in the same cell. This approach is obviously validated by the good agreement shown in Fig. 4 at small radii. 


\section{Ly- $\alpha$ during the EoR}

\subsection{The role of $L y-\alpha$ photons in the $21 \mathrm{~cm}$ emission}

The 21-cm signal can be seen in emission or absorption against the CMB. The differential brightness temperature observed at redshift $z=0$ is

$\delta T_{\mathrm{b}}=\frac{T_{\mathrm{S}}-T_{\mathrm{CMB}}}{1+z}\left(1-\mathrm{e}^{-\tau_{21}}\right)$,

where $T_{\mathrm{S}}$ is the neutral hydrogen spin temperature, $T_{\mathrm{CMB}}$ the CMB radiation blackbody temperature at redshift $z$, and $\tau_{21}$ the $21-\mathrm{cm}$ line optical depth. The value of $\tau_{21}$ is given, among others, by Tozzi et al. (2000) or Furlanetto et al. (2006a). Injecting the formula for $\tau_{21} \ll 1$, the differential brightness temperature can be written as

$\delta T_{\mathrm{b}} \sim 9 . x_{\mathrm{HI}}(1+\delta)(1+z)^{\frac{1}{2}} \frac{T_{\mathrm{S}}-T_{\mathrm{CMB}}}{T_{\mathrm{S}}} \mathrm{mK}$,

where $\delta$ is the local overdensity at redshift $z$, and $x_{\mathrm{HI}}$ the neutral hydrogen fraction. This value of $\delta T_{\mathrm{b}}$ is for a flat $\Lambda \mathrm{CDM}$ model with $h_{0}=0.7$ and $\Omega_{\mathrm{b}}=0.044$. It changes by $\pm 0.5 \mathrm{mK}$ when $\Omega_{\mathrm{m}}$ varies from 0.25 to 0.3 . The value of the spin temperature $T_{\mathrm{S}}$ is the result of three competing processes. The absorption/reemission of CMB photons tends to bring $T_{\mathrm{S}}$ to $T_{\mathrm{CMB}}$ over a time scale under $10^{5}$ years during the EoR (Tozzi et al. 2000). As we have seen in the Introduction, both collisions between hydrogen atoms and the pumping by Ly- $\alpha$ photons, also known as the Wouthuysen-Field effect (Woutuysen 1952; Field 1958), instead tends to couple $T_{\mathrm{S}}$ to the kinetic temperature of the gas. As a result, the spin temperature can be written (Furlanetto et al. 2006a) as

$T_{\mathrm{S}}^{-1}=\frac{T_{\mathrm{CMB}}^{-1}+x_{\mathrm{c}} T_{\mathrm{K}}^{-1}+x_{\alpha} T_{\mathrm{C}}^{-1}}{1+x_{\mathrm{c}}+x_{\alpha}} \quad$ with $\quad T_{\mathrm{C}} \simeq T_{\mathrm{K}}$,

where $x_{\mathrm{c}}$ and $x_{\alpha}$ are the coupling coefficients for collisions and Ly- $\alpha$ pumping respectively, and $T_{\mathrm{C}}$ is the effective color temperature of the UV radiation field (see Furlanetto et al. 2006a). The coefficient $x_{\alpha}$, which is the focus of this work, can be explicitly written as

$x_{\alpha}=\frac{4 P_{\alpha} T_{\star}}{27 A_{10} T_{\mathrm{CMB}}}$,

where $T_{\star}=0.068 \mathrm{~K}, A_{10}=2.85 \times 10^{-15} \mathrm{~s}^{-1}$ is the spontaneous emission factor of the $21-\mathrm{cm}$ transition, and $P_{\alpha}$ is the number of scatterings of Ly- $\alpha$ photons per atom per second. Now, two approximations usually occur that we do not make in this paper. The first is that $P_{\alpha}$ is considered proportional to $J\left(v_{\alpha}\right)$, the angle averaged specific intensity at the local Ly- $\alpha$ frequency, neglecting the contribution of wing absorptions. We will see that this approximation is valid, provided $J(v)$ itself has been computed taking wing absorptions into account. The second, more drastic approximation is to evaluate $J(v)$ without performing the full radiative transfer computation. Actually, to our knowledge, all numerical simulations of 21-cm emission consider a uniform value for $J\left(v_{\alpha}\right)$. However, Barkana \& Loeb (2005) have shown that several factors induce fluctuations in $J\left(v_{\alpha}\right)$ : the $1 / r^{2}$ scaling of the flux that magnifies the Poisson noise in the source distribution, the clustering of the sources, and the contribution of higher Ly- $\alpha$ series photons (also studied in detail by Pritchard \& Furlanetto 2006). They predict the power spectrum of the 21-cm brightness temperature due to the fluctuation in $J\left(v_{\alpha}\right)$. Although this is a vast improvement over using a uniform Ly- $\alpha$ flux, they are still neglecting radiative transfer effects in

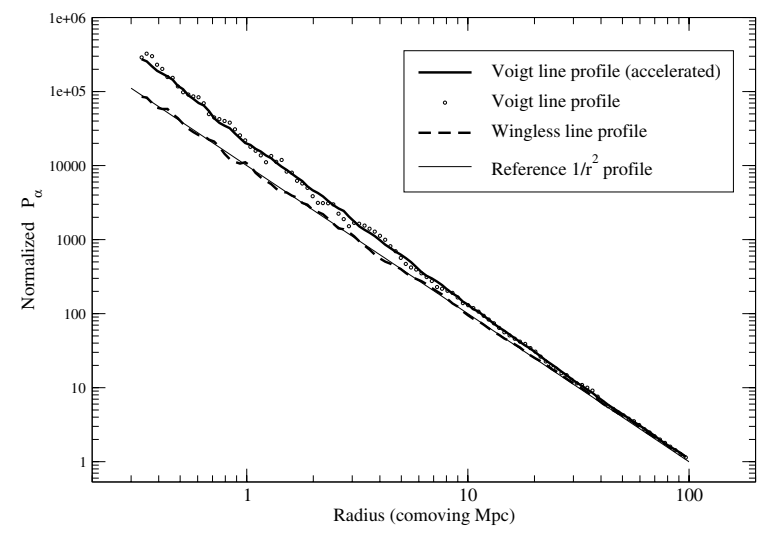

Fig. 5. Profiles of the scattering rate per atom, $P_{\alpha}$, in the Ly- $\alpha$ line, around a central source with a continuous flat spectrum in a homogeneous medium of neutral hydrogen at $30 \mathrm{~K}$ and the average baryon density of the universe at $z \sim 10$.

assuming that photons are freely streaming until they redshift to the local Ly- $\alpha$ frequency. We show that this assumption breaks down at scales smaller that $\sim 10$ comoving Mpc.

Potentially, pumping the upper excitation level is not the only way for Ly- $\alpha$ photons to influence the $21-\mathrm{cm}$ emission: they also heat up the gas. This heating mechanism was first thought to be efficient by Madau et al. (1997). However, Chen \& Miralda-Escudé (2004), taking the atoms' thermal velocity distribution into account that had been neglected by Madau et al., found a much lower, actually negligible, heating rate. Furlanetto \& Pritchard (2006), considering the effect of higher Lyman series photons, confirmed this result and found, for typical EoR conditions, the Ly- $\alpha$ heating rate to be 140 lower than the heating rate from X-rays. Chuzhoy \& Shapiro (2007) recently challenged this result, in particular adding the effect of the deuterium Ly- $\beta$ resonance line, but the strength of this effect is not yet completely probed.

\section{2. $P_{\alpha}$ profiles for spherically symmetric configurations}

In the next three cases, we consider a central source in a spherically symmetric medium of neutral hydrogen at $T_{\mathrm{K}}=30 \mathrm{~K}$ and $z \simeq 10$, which is typical of the EoR. The main difference with the setup of the tests in Sect. 4.3 is that the source emits a flat continuous spectrum. We only deal with photons between Ly- $\alpha$ and $\mathrm{Ly}-\beta$ frequencies.

\subsubsection{Homogeneous medium}

First, we consider the case where the gas is homogeneous with a density equal to the average density of the universe at $z \sim 10$. We ran the simulation in a 600 comoving Mpc box with the source in the center of the box. We present the results up to a radius of 100 comoving Mpc. At larger radii, the effect of the box boundaries (no retrodiffusion) and the variation in $a$ during the photon flight (see Sect. 2.1.3) alter the validity of the simulation. Figure 5 presents the radial profile of the scattering rate per atom, $P_{\alpha}$, in different cases.

Using the real Voigt line profile, we can see a deviation at small radii from the simple $1 / r^{2}$ profile expected if photons stream freely from the source until they redshift to the local Ly- $\alpha$ frequency. Here is why. Let us consider a photon emitted above the Ly- $\alpha$ frequency. As it travels away from the source, it is redshifted toward the local Ly- $\alpha$ frequency. Because of the contribution of the wings of the Voigt profile to the optical depth, it 
has a probability of scattering before reaching the local Ly- $\alpha$ frequency. On average, it will scatter for the first time when the optical depth along its path reaches 1 . This is achieved when the photon is redshifted to the frequency $\sim v_{\alpha}+v_{\star}$ (see Sect. 4.3), which occurs at a distance $r_{\star}$ before the location where it would reach the local Ly- $\alpha$ frequency (quantities defined in Loeb \& Rybicki 1999). In our setup, typical of the EoR, $r_{\star} \sim 10$ comoving Mpc. After the photons scatter for the first time, they change direction. Consequently, the location where a photon actually enters the core of the local Ly- $\alpha$ line has a probability of being anywhere within $\sim 10$ comoving Mpc of the location determined by free streaming alone. This is not crucial on large scales where the expected $1 / r^{2}$ profile is recovered, but it creates a steeper profile on small scales (exponent $\sim-2.3$ in our setup). To validate our interpretation, we computed the transfer with a modified line profile: the core of the line is unchanged, but the wings are set to zero. As can be seen in Fig. 5, we then recover the $1 / r^{2}$ profile. After this paper was submitted, Chuzhoy \& Zheng (2007) posted a paper with a similar result. They considered a very similar setup and computed the transfer with a simple Monte Carlo code that is 1-D (spherical symmetry) and uses a simplified line profile but does include Ly- $\alpha$ photons locally injected by cascades from upper Lyman series lines. They find the same steepening of the scattering rate profile on short scales. However, they show that photons injected from upper Lyman series lines are much less sensitive to radiative transfer effect, and they follow the $1 / r^{2}$ profile more closely. Consequently, the discrepancy between the full radiative transfer computation and the simple $1 / r^{2}$ evaluation is somewhat reduced.

Finally we checked the effect of using the core-skipping acceleration scheme for evaluating the fluctuations of $P_{\alpha}$ : since all core absorptions are avoided, it could have modified the spatial $P_{\alpha}$ fluctuation map. As can be seen in Fig. 5, this is not the case. Although the wings modify the location where photons enter the core, the scattering number is still dominated by the close-to-the-core contribution.

\subsubsection{Central clump}

Until now, the Ly- $\alpha$ scattering rate has been evaluated during the EoR only in a homogeneous medium (Barkana \& Loeb 2006; Pritchard \& Furlanetto 2006). With a general-purpose 3D code such as LICORICE, we can investigate the impact of density fluctuations in the gas on the Ly- $\alpha$ scattering rate per baryon. First we choose a very simple setup by considering a box of size 64 comoving Mpc, with a central homogeneous spherical clump of gas of radius 1 comoving Mpc. The clump is 64 times denser than the surrounding medium, which has the average baryon density of the universe at the redshift of the simulation $(z \sim 10)$. The source is in the center of the clump and has a continuous flat spectrum. The radial profile of the Ly- $\alpha$ scattering rate per atom, $P_{\alpha}$, is shown in Fig. 6. The main feature is a depletion of $P_{\alpha}$ in the low-density medium just outside the clump. Indeed, photons that should redshift to the Ly- $\alpha$ frequency in this region first have to travel through the high-density region where they have an enhanced probability of being scattered in the wing of an atom and redirected to redshift to local Ly- $\alpha$ while still inside the core. In other words, the enhanced wing scatterings in the clump draw core scatterings from surrounding regions to the clump itself. This is somewhat similar to the usual shadowing effect in radiative transfer, although in this case the process occurs in the frequency space, so the shadowing can be seen even in a spherical configuration. Obviously, in addition to the $1 / r^{2}$ decline of the flux and the fluctuations in the source distribution,

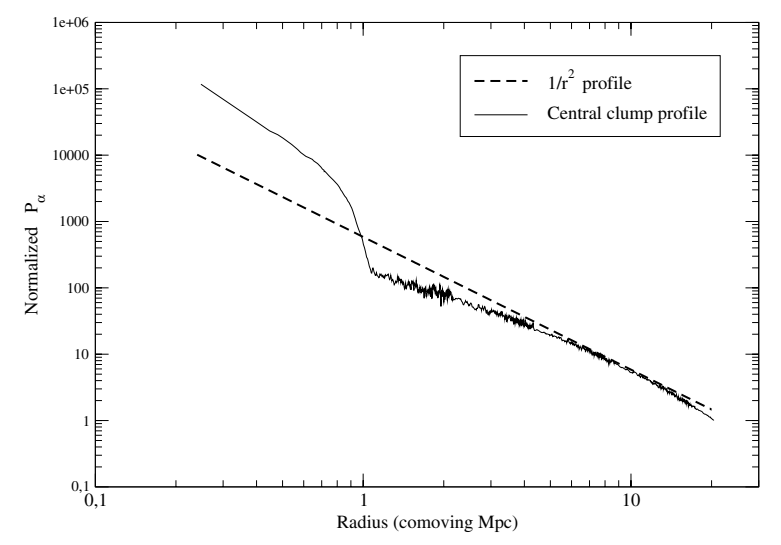

Fig. 6. Profile of the scattering rate per atom, $P_{\alpha}$, in the Ly- $\alpha$ line, around a central source with a continuous flat spectrum, inside an overdense spherical clump of gas (64 times the density of the surrounding medium) of radius 1 comoving Mpc.

we can expect a new source of fluctuations for $P_{\alpha}$ : the density fluctuations of the intergalactic medium.

\subsubsection{Isothermal density profile}

Instead of the sharp density transition between the clump and the surrounding medium we now consider an isothermal density profile $\left(\rho \sim 1 / r^{2}\right)$ around the source. The isothermal profile connects to the surrounding homogeneous medium at a radius of 10 comoving Mpc. All other parameters of the simulation are the same as in the previous setup. The radial profile of the Ly- $\alpha$ scattering rate per atom, $P_{\alpha}$, is shown in Fig. 7. Since the average density is higher than in the other setups, photons scatter many times and even with a acceleration method, we used only $2 \times 10^{4}$ photons. To avoid a very high noise level at large radii, we used an adaptative resolution: high in the center, lower in the outer regions. We can see in the figure that, inside the region with an isothermal density profile, $P_{\alpha}$ matches a $1 / r^{3}$ profile closely. It reverts to $1 / r^{2}$ in the homogeneous medium outside the $10 \mathrm{Mpc}$ radius. This shows that the brightness temperature fluctuations of the $21-\mathrm{cm}$ emission may be stronger than previously estimated on small scales, at least during the early EoR when $P_{\alpha}$ fluctuations are meaningful.

Is this setup more effective in predicting $P_{\alpha}$ fluctuations during the EoR than a uniform medium? Yes, in the sense that the medium should obviously be denser closer to the source. However the spherical symmetry and the specific profile used here are oversimplified, since the actual intergalactic medium in the EoR is not in an equilibrium configuration, especially during this early period of sources formation, and the spherical symmetry is broken by the presence of filaments. In the next section we investigate how filaments modify $P_{\alpha}$.

\section{3. $P_{\alpha}$ map for an axisymmetric configuration}

We now consider an axisymmetric density field for the gas. Inside a cylinder of radius 1 comoving Mpc, the density is 64 times the critical baryon density, while outside it is equal to the critical baryon density. The source is located on the symmetry axis. All other parameters are identical to the previous setup. This new setup is a simplified representation of the real case of source formation during the EoR at the intersection of several filaments, where the filaments have a density profile. The $P_{\alpha}$ contour map is shown in Fig. 8. The shaded area represents 


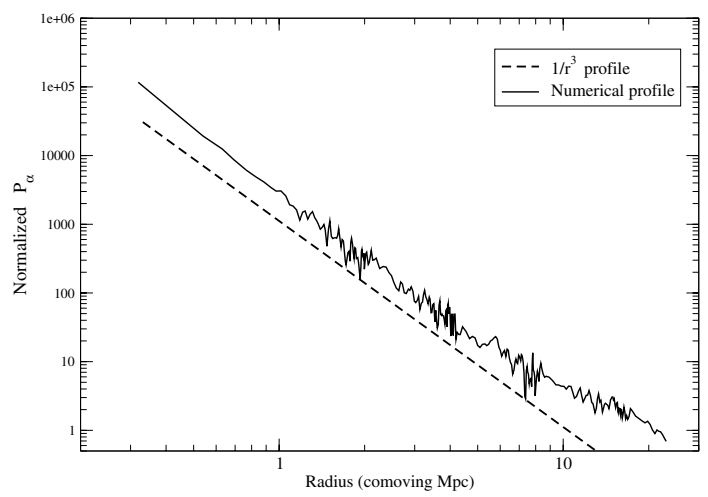

Fig. 7. Profile of the scattering rate per atom $P_{\alpha}$ in the Ly- $\alpha$ line, around a central source with a continuous flat spectrum. Inside a sphere of radius 10 comoving $\mathrm{Mpc}$, the gas density field is an isothermal sphere, while outside it is homogeneous at the average baryon density of the universe $(z \sim 10)$. The two regions connect smoothly. A $1 / r^{3}$ profile is plotted for comparison.

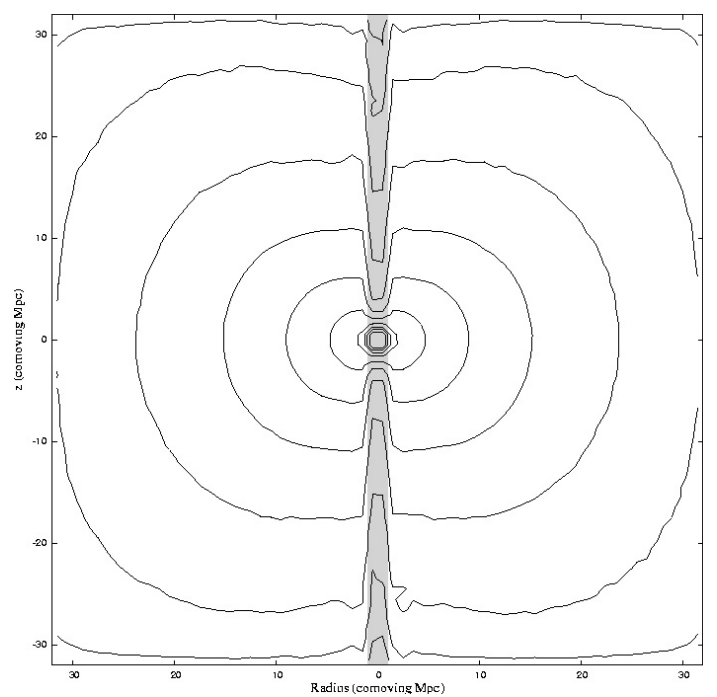

Fig. 8. Contours of the scattering rate per atom $P_{\alpha}$ in the Ly- $\alpha$ line, around a central source with a continuous flat spectrum. The source is located inside a cylindrical homogeneous filament of gas (shaded zone in the figure) with a density 64 times the density of the surrounding medium, which is at the average baryon density of the universe $(z \sim 10)$. The contours are equally spaced on a logarithmic scale with a step of $\sqrt{10}$.

the filament, and the contours are equally spaced on a logarithmic scale (two per decade). The map is integrated over a variation of $\pi$ in the angular variable. About $5 \times 10^{6}$ photons were used for this simulation. There is some boundary effect due to the finite size of the simulation box: photons that would scatter just outside the simulation box and possibly reenter the box are lost instead. This affects a few Mpc near the boundary. We can see a sharp depletion in the number of scatterings per atom inside the filament. We have checked that a lower density contrast creates a smaller depletion, as expected. Once again, we see that the gas density field fluctuations induce fluctuations in $P_{\alpha}$. However, the weaker Wouthuysen-Field effect in denser regions may be balanced by the greater coupling due to collisions (which is proportional to the density). What may be more relevant to the future observations than what occurs on small scales inside the filaments is that the presence of the filaments modifies the shape of the contours in the low-density surrounding medium: they are

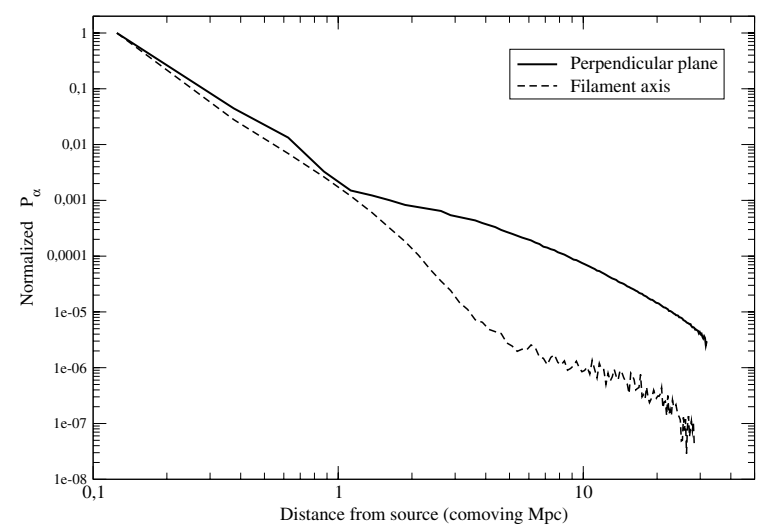

Fig. 9. Scattering rate per atom $P_{\alpha}$ in the Ly- $\alpha$ line along the axis of a filament and in a perpendicular plane. A central source with a continuous flat spectrum is located at the intersection of the filament and the plane. The filament is cylindrical with radius 1 comoving Mpc and homogeneous with a density 64 times the density of the surrounding medium, which is at the average baryon density of the universe $(z \sim 10)$.

not spherical but oblate. With our density contrast, the axis ratio is about 2 in the $5-10 \mathrm{Mpc}$ range and decreases at large distances. Figure 9 shows the normalized $P_{\alpha}$ profiles along filament axis and in the perpendicular plane containing the source. While inside the filament (distance smaller than 1 comoving $\mathrm{Mpc}$ ), the profiles match. At larger distances, Fig. 9 quantifies the $P_{\alpha}$ ratio between the two regions: it reaches a maximum value of $\sim 100$ at a distance of $\sim 5 \mathrm{Mpc}$.

\section{Conclusions}

The main goal of this paper was to investigate a source of fluctuations in the brightness temperature of the $21-\mathrm{cm}$ emission during the EoR, which is usually neglected in numerical simulations. The Wouthuysen-Field effect, which couples the hydrogen spin temperature to the kinetic temperature of the gas, is regulated by the crucial parameter $P_{\alpha}$, the number of scatterings per atom per second. While all previous simulations of the 21-cm emission used a uniform value for $P_{\alpha}$, Loeb \& Barkana (2005) have shown, in a simple theoretical framework, that several sources of fluctuations in $P_{\alpha}$ can modify the power spectrum of the $21-\mathrm{cm}$ emission. We studied how a full 3D radiative transfer treatment of the Ly- $\alpha$ line in a cosmological context modifies the picture given by Loeb and Barkana.

The first step was to implement and validate the Ly- $\alpha$ radiative transfer in LICORICE. We used a Monte-Carlo approach, and implemented an algorithm and acceleration schemes along the same lines as those of other existing codes. We discarded physical processes such as recoil or deuterium contribution, which have negligible effects on the Ly- $\alpha$ transfer during the EoR. On the other hand we accurately computed the optical depth in an expanding cosmological medium without any resolution effects. We presented three validation tests for the code. The first two are classical setups: a monochromatic source in a static uniform sphere of gas and in an expanding sphere of gas. The agreement for the emerging spectrum with analytic solutions and results by other authors is good. The third test, the mean intensity map for a monochromatic source in an expanding sphere of gas at $T=0 \mathrm{~K}$, focuses on a quantity that is more closely related to $P_{\alpha}$. The comparison with the analytic solution provided by Loeb and Rybicki is good where the analytic solution is valid: in the diffusion regime. This set of tests strongly suggests that LICORICE is valid. 
Barkana \& Loeb (2005) show that several factors contribute to the fluctuations in the local Ly- $\alpha$ flux: the $1 / r^{2}$ scaling of the flux and the Poisson noise and clustering in the sources distribution. They assume, however that photons redshift freely from the source until they reach the local Ly- $\alpha$ frequency and only then scatter off hydrogen atoms. In other words, they neglect wing scattering. We computed the $P_{\alpha}$ profile for a source with a flat continuous spectrum in a uniform expanding medium. We showed that the effect of taking the wing scatterings into account in a full radiative transfer code is to steepen the profile on small scales to $\mathrm{a} \sim 1 / r^{2.3}$ profile (with our choice of parameters). On large scales, or when wings are suppressed, we recover the $1 / r^{2}$ profile. Thus we may expect, on small scales, more power in the $21-\mathrm{cm}$ emission than predicted by Barkana \& Loeb. But a still stronger effect was obtained when we introduced fluctuations in the density of the gas surrounding the source. We investigated a central clump setup and an isothermal profile. In both cases, we observed alterations in the $P_{\alpha}$ profile. In the case of an isothermal density profile, we found a $\sim 1 / r^{3}$ profile for $P_{\alpha}$. This also suggests stronger fluctuations than predicted by Barkana \& Loeb but, once again, mainly on small scales since the $\sigma_{8}$ at $z=10$ is only $\sim 0.1$. Large scale structures had not grow much yet.

Finally, we tried to create a more realistic situation by placing a flat spectrum source inside a filament of overdense gas. We observed a sharp depletion of $P_{\alpha}$ inside the filament. The photons scatter out of the filament before they reach the core of the line. This fluctuation inside the filament is once again a small-scale feature, so it may be difficult to catch with LOFAR or SKA. However, the presence of the filament also produced oblate contours for $P_{\alpha}$ at larger scales ( $>10$ comoving Mpc), in the surrounding medium. This effect may be more within reach of the resolution of these instruments.

The increased fluctuations of $P_{\alpha}$ due to radiative transfer effects and to the inhomogeneous distribution of the gas translate linearly into fluctuations of the $x_{\alpha}$ coefficient. On one hand, we expect these fluctuations to be globally significant and produce brightness temperature fluctuations only as long as $x_{\alpha} \sim 1$, i.e. as long as the coupling does not saturate to $T_{\mathrm{s}}=T_{\mathrm{K}}$. This occurs in the early phase of reionization. It is not possible to be much more specific in terms of redshift because the Ly- $\alpha$ pumping efficiency depends strongly on the model for the source type and formation history. On the other hand, we have shown that density fluctuations in the gas can create fluctuations of $P_{\alpha}$ of a factor greater than 10 . The depleted regions will fill up only when the amount of young sources gets more than 10 times larger. Cosmological simulations suggest that this corrresponds to a change in redshift between 1 and 2 around redshift 10. Consequently, we expect that the fluctuations of $P_{\alpha}$ due to inhomogeneous gas will lead to a longer survival of depleted regions where $T_{\mathrm{S}}$ remains coupled to $T_{\mathrm{CMB}}$. One may argue that the strongest fluctuations of the gas density are located around the sources and are ionized very early. However, numerical simulations have shown that the ionization front is not spherical but that it is trapped in the high density regions such as filaments pointing to the source, where reionization is delayed a lot (see for example, Gnedin 2000). As a result, these filaments should be able to play their role in creating $P_{\alpha}$ fluctuations.

We have not investigated the effect on $P_{\alpha}$ of an anisotropic peculiar velocity field around the source. In principle, it would also induce non spherical contours. However, cosmological simulations in a $20 \mathrm{Mpc}$ box suggest peculiar velocities on the order of $100 \mathrm{~km} \mathrm{~s}^{-1}$ during the EoR, when the Hubble constant is $\mathrm{H}(z=10) \sim 1000 \mathrm{~km} \mathrm{~s}^{-1} \mathrm{Mpc}^{-1}$. Moreover, only velocity differences will alter $P_{\alpha}$ contours, which should be smaller than $100 \mathrm{~km} \mathrm{~s}^{-1}$ on large scales. We estimate that the impact of the velocity field of the gas is smaller than the impact of the density fluctuations. However, LICORICE fully implements the effects of the gas peculiar velocity and it will be taken into account in the future simulations of a cosmological box.

Another process will have to be included in the future: the effect of higher Lyman series lines. Barkana \& Loeb (2005) and Pritchard \& Furlanetto (2006) have shown that these lines, which have horizons closer to the source than the Ly- $\alpha$ line, add to the power of the $P_{\alpha}$ fluctuations close to the sources. In a forthcoming paper, we will apply LICORICE to a cosmological field during the EoR, using simulation outputs from the HORIZON project $^{2}$, and compute the resulting 21-cm brightness temperature map. If, as we believe, we find significant modification of the predictions, we will include higher Lyman series lines.

Acknowledgements. This work was realized in the context of the SKADS and HORIZON projects.

\section{References}

Abel, T., Norman, M. L., \& Madau, P. 1999, ApJ, 523, 66 Ahn, S.-H., Lee, H.-W., \& Lee, H. M. 2001, ApJ, 554, 604 Ahn, S.-H., Lee, H.-W., \& Lee, H. M. 2002, ApJ, 567, 922 Avery, L. W., \& House, L. L. 1968, ApJ, 152, 493

Barkana, R., \& Loeb, A. 2005, ApJ, 626, 1

Carilli, C. L. 2006, NewAR, 50, 162

Carilli, C. L., Gnedin, N., Furlanetto, S., \& Owen, F. 2004, NewAR, 48, 1053

Chen, X., \& Miralda-Escudé, J. 2004, ApJ, 602, 1

Chuzhoy, L., \& Shapiro, P. R. 2007, ApJ, 655, 843

Chuzhoy, L., \& Zheng, Z. 2007 [arXiv:astro-ph/0706.0895]

Ciardi, B., \& Madau, P. 2003, ApJ, 596, 1

Ciardi, B., Scannnapieco, E., Stoehr, F., et al. 2006, MNRAS, 366, 689

Cantalupo, S., Porciani, C., Lilly, S., \& Miniati, F. 2005, ApJ, 628, 61

Dijkstra, M., Haiman, Z., \& Spaans, M. 2006, ApJ, 649, 14

Fan, X., Carilli, C. L., \& Keating, B. 2006, ARA\&A, 44, 415

Field, G. B. 1958, Oric. IRE, 46

Furlanetto, S. R. 2006, MNRAS, 371, 867

Furlanetto, S. R., \& Pritchard, J. R. 2006, MNRAS, 372, 1093

Furlanetto, S. R., Sokasian, A., \& Hernquist, L. 2004, MNRAS, 347, 187

Furlanetto, S. R., Oh, S. P., \& Briggs, F. H. 2006, PhR, 433, 181

Gnedin, N. Y. 2000, ApJ, 535, 530

Gnedin, N. Y., \& Shaver, P. A. 2004, ApJ, 608, 611

Hansen, M., \& Oh, P. 2006, MNRAS, 367, 979

Iliev, I. T., Shapiro, P. R., \& Raga, A. C. 2005, MNRAS, 361, 405

Iliev, I. T., Mellema, G., Pen, U.-L., et al. 2006a, MNRAS, 369, 1625

Iliev, I. T., Ciardi, B., Alvarez, M., et al. 2006b, MNRAS, 371, 1057

Iliev, I. T., Mellema, G., Pen, U.-L., Bond, J. R., \& Shapiro, P. R. 2007 [arXiv: astro-ph/0702099]

Kuhlen, M., Madau, P., \& Montgomery, R. 2006, ApJ, 637, L1

Loeb, A., \& Rybicki, G. 1999, ApJ, 524, 527

Maselli, A., Ferrara, A., \& Ciardi, B. 2003, MNRAS, 345, 379

McQuinn, M., Lidz, A., Zahn, O., et al. 2007, MNRAS, 377, 1043

Madau, P., Meiksin, A., \& Rees, M. J. 1997, ApJ, 475, 429

Mellema, G., Iliev, I. T., Pen, U.-L., \& Shapiro, P. R. 2006, MNRAS, 372, 679

Neufeld, D. A. 1990, ApJ, 350, 216

Nusser, A. 2005, MNRAS, 359, 183

Peebles, P. J. E. 1993, Principles of Physical Cosmology (Princeton Univ. Press), 573

Pritchard, J. R., \& Furlanetto, S. R. 2006, MNRAS, 367, 1057

Semelin, B., \& Combes, F. 2002, A\&A, 388, 826

Shapiro, P. R., Ahn, K., Alvarez, M. A., et al. 2006, ApJ, 646, 681

Spergel, D. N., Bean, R., Dore, O., et al. 2007, ApJS, 170, 377

Tasitsiomi, A. 2006, ApJ, 645, 792

Tozzi, P., Madau, P., Meiksin, A., \& Rees, M. 2000, ApJ, 528, 597

Verhamme, A., Schaerer, D., \& Maselli, A. 2006, A\&A, 460, 397

Valdès, M., Ciardi, B., Ferrara, A., Johnston-Hollitt, M., \& Röttgering, H. 2006, MNRAS, 369, L66

Wouthuysen, S. A. 1952, AJ, 57, 31

Zheng, Z., \& Midalda-Escudé, J. 2002, ApJ, 578, 33

\footnotetext{
${ }^{2}$ http://www .projet-horizon.fr
} 